Article

\title{
Surface Tension-Based Alignment of Microfibers on Hydrophilic-Superhydrophobic Grooved Surfaces
}

\author{
Bo Chang ${ }^{1, *}$, Jialong Jin ${ }^{1}$ and Quan Zhou ${ }^{2}$ \\ 1 College of Mechanical and Electrical Engineering, Shaanxi University of Science and Technology, \\ Xi'an 710021, China; 1905004@sust.edu.cn \\ 2 School of Electrical Engineering, Aalto University, FI-00076 Aalto, Finland; quan.zhou@aalto.fi \\ * Correspondence: changbo@sust.edu.cn
}

Received: 7 October 2020; Accepted: 29 October 2020; Published: 29 October 2020

\begin{abstract}
Alignment and orderly distribution of microfibers have a major effect on the mechanical, electrical, and thermal properties of the fiber reinforced materials, biomimetic materials, and soft microsensors. However, it is still a challenging task to precisely align and distribute microfibers and construct complex patterns. This paper proposes a surface tension-based method to align and orderly distribute microfibers. A model was developed to simulate the surface tension driven alignment of the microfiber. We designed and fabricated hydrophilic-superhydrophobic grooved surfaces. We demonstrated that the microfibers can self-align to the hydrophilic-superhydrophobic grooves with different geometries. We studied the influence of the volume of the droplet and bias on the alignment success rate. The results indicate that the process can tolerate large variations of the bias and the volume, unless the volume is not enough to cover the groove. We further investigated the influence of the width of the groove on the alignment accuracy. The results show that the alignment accuracy is largely depending on the size difference between the groove and the microfiber; the better the size of the groove matches the size of the fiber, the higher the alignment accuracy will be achieved. The proposed method has great potential in construction of complex microstructures using microfibers.
\end{abstract}

Keywords: micro assembly; self-alignment; surface tension; hydrophilic-superhydrophobic; microfibers; orderly distribution; robotic micro assembly; micro manipulation; micro groove

\section{Introduction}

The distribution and the alignment of microfibers have a major effect on the mechanical [1], electrical [2], and thermal properties [3] of the fiber reinforced materials. Polymers with aligned carbon nanotubes have shown much higher conductivity and tensile strength compared to the polymer with non-aligned carbon nanotubes [4]. It has been reported that the tensile strength of an epoxy composite with aligned carbon nanotubes has improved from $8 \mathrm{MPa}$ to $21.1 \mathrm{MPa}$ and Young's modulus from $415 \mathrm{MPa}$ to $843 \mathrm{MPa}$ [5]. It has been also shown that the electrical resistivity of aligned nanotubes was reduced by one order of magnitude compared to non-aligned nanotubes [6].

Many methods have been developed to align microfibers and form certain patterns, including electrospinning [7-11], 3D printing [12-14], robotic micromanipulation [15-17], microfluidics [18,19], and so on. Electrospinning uses electric force to dispense charged threads of polymer solutions or melt polymers and the method is especially suitable for producing exceptionally fine fibers such as micro and nanofibers. Distribution and alignment of micro- and nanofibers have been previously demonstrated using the electrospinning method [8,13], e.g., fibers with a certain geometry such as lattice pattern [13] and ivy shoot like patterns [20] have been achieved by adjusting the concentration of the polymer solution, flow rate, and electric field. A distribution of microfibers with the orientation 
of $90^{\circ}$ and $60^{\circ}$ has been achieved by combining 3D printing technique and melt electrospinning technique [7]. Microfluidics and robotic micromanipulation technology have also been reported to be able to align microfibers and construct simple structures, e.g., small amount of the microfibers can be distributed in parallel or orthogonally $[18,19]$. Despite the impressive results achieved so far, it is still challenging to precisely control the spacing of two individual microfibers and the orientation of each microfiber and construct more complex patterns. Previously, our research group has reported a hybrid microassembly technique [21-25] which combines the robotic micro-assembly technique and the surface tension-based self-alignment technique [26-30]. The hybrid microassembly technique has been demonstrated to be able to achieve accurate and fast alignments of microchips, achieving the assembly of 40,000 microchips per hour, with an accuracy higher than $1 \mu \mathrm{m}$, and a success rate of $98 \%$ [31]. The hybrid microassembly method has shown good potentials for construction of 3D microstructures; however, the method has not been investigated for the alignment of microfibers.

In this paper, we propose a surface tension-based method to align and orderly distribute microfibers. To understand the mechanism, a theoretical model was developed to simulate the alignment of the microfiber and analyze the driven force for the alignment of microfibers. We designed and fabricated grooved hydrophilic-superhydrophobic patterned surfaces. We demonstrated that the microfibers can self-align to the grooved hydrophilic-superhydrophobic patterned surfaces with different geometries. We studied the influence of the volume of the droplet and bias on the alignment success rate through systematic experiments. We further investigated the influence of the width of the grooved pattern on the alignment accuracy.

\section{Materials and Methods}

\subsection{Alignment Strategy}

In this paper, we propose a surface tension-based alignment method which combines surface tension assisted pick-and-place technique and hydrophilic-superhydrophobic grooved surfaces. It utilizes the surface tension assisted pick-and-place technique for the coarse positioning of the flexible fibers and applies hydrophilic-superhydrophobic grooved surfaces to achieve fine alignment of microfibers. The schematic of the alignment method is illustrated in Figure 1. Firstly, a microfiber is picked up by a dispensing needle with a droplet (Figure 1a); next, the fiber is transported to a target hydrophilic-superhydrophobic grooved surface (Figure 1b); and then the needle dispenses a drop of water and the water droplet is confined inside the hydrophilic groove and form a water meniscus (Figure 1c); next, the fiber is released from the needle and aligned to the target groove (Figure 1d); finally, the water droplet inside the groove evaporates leaving the fiber aligned to the groove (Figure 1e).

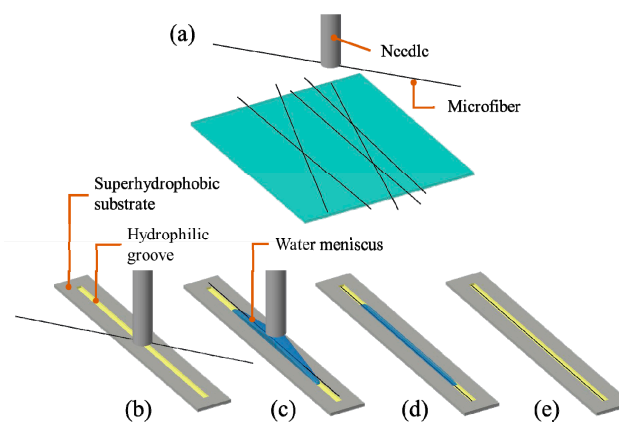

Figure 1. Schematic of surface tension-based alignment of a microfiber: (a) A microfiber is picked up by a needle; (b) The fiber is transport it to a target hydrophilic-superhydrophobic grooved surface; (c) The needle dispenses a drop of water and the water droplet is confined inside the hydrophilic groove; (d) The fiber is released from the needle and aligned to the groove; (e) The water droplet inside the groove evaporates. 


\subsection{Fabrication of Hydrophilic-Superhydrophobic Grooved Surfaces}

To fabricate hydrophilic-superhydrophobic grooved surfaces for alignment of microfibers, superhydrophobic coating (WHOLE-NANO SPN-62, WHOLE-NANO Ltd, Suzhou, China) was firstly sprayed on a $10 \mathrm{~mm}$ (length) $\times 10 \mathrm{~mm}$ (width) $\times 0.5 \mathrm{~mm}$ (thickness) silicon substrate and dried at room temperature, then an ultraviolet laser cutting machine LU-5 (HGTECH, Power of 5 W, Wavelength of $355 \mathrm{~nm}$, Huagong Tech. Ltd, Wuhan, China) was used to fabricate microgrooves on the silicon substrate. The laser cutting machine was operated at the speed of $2000 \mathrm{~mm} / \mathrm{s}$, with the frequency of $100 \mathrm{kHz}$, the pulse width of $1 \mu \mathrm{s}$, and the current of $1 \mathrm{~A}$. The width of the fabricated grooves was in the range of $100 \mu \mathrm{m}-500 \mu \mathrm{m}$, the length of the groove was $4 \mathrm{~mm}$, and the depth of the groove was around $35 \mu \mathrm{m}$. Microfibers used for the alignment test are glass fibers with a diameter of $13 \mu \mathrm{m}$ and a length of $3.7 \mathrm{~mm}-4 \mathrm{~mm}$. Figure 2 shows the glass microfiber and the fabricated grooved silicon substrate. Figure 2a,b represents the microscopic view of the glass fiber, and the diameter of the glass fiber is around $13 \mu \mathrm{m}$, which is about one fifth of a human hair in diameter. Figure $2 \mathrm{c}$ shows the fabricated grooved silicon substrate, where the size of the grooves is $4 \mathrm{~mm}$ (length) $\times 0.5 \mathrm{~mm}$ (width) $\times 0.035 \mathrm{~mm}$ (thickness). Figure $2 \mathrm{~d}$ shows an image of the water contact angle $\left(155^{\circ}\right)$ on the superhydrophobic substrate. Figure $2 \mathrm{e}$ shows that the water contact angle in the groove is less than $5^{\circ}$.
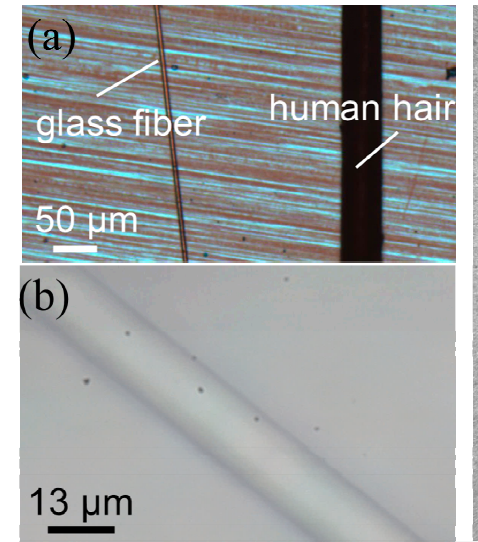

(d) Superhydrophobic substrate Contact angle: $155^{\circ}$

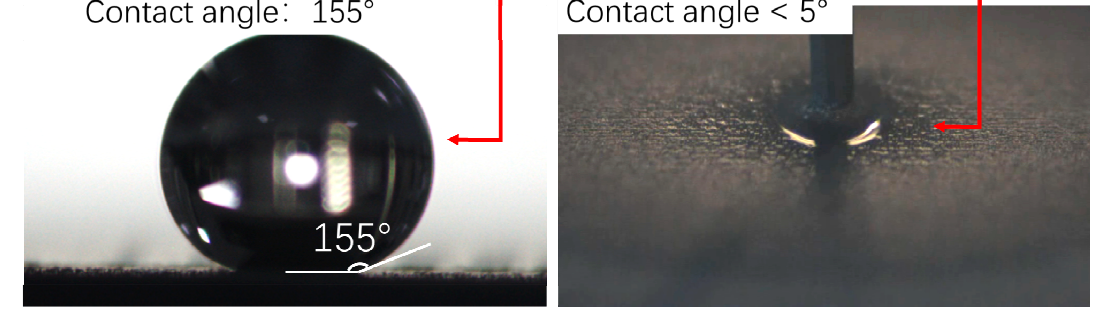

Figure 2. Glass fiber and grooved silicon substrate: (a) Microscopic view of a glass fiber next to a human hair; (b) Microscopic view of glass fiber with a diameter of $13 \mu \mathrm{m}$; (c) Hydrophilic-superhydrophobic patterned substrate with five parallel grooves; (d) Contact angle of water on the superhydrophobic substrate; (e) Contact angle of water in the hydrophilic groove.

\subsection{Experimental Setup}

To align microfibers using surface tension-based alignment method, a robotic system has been set up as shown in Figure 3. The system consisted of a vision system, a needle dispensing system, and a sample carrier. The alignment process was observed both from the tilted view and from the side with a vision system consisting of two microscopes with cameras (Point Grey BFLY-U3-23SS6C and Point Grey GS3-U3-23S6M-C, Edmund Optics, Barrington, IL, USA). The needle dispensing system was attached to a Cavro Centris pump (Tecan Group Ltd, Männedof, Switzerlan) and used to generate nanoliter water droplets on the hydrophilic-superhydrophobic grooved surfaces. The inner diameter of the needle was 
$160 \mu \mathrm{m}$. The sample carrier was built by combining three motorized stages (two M-122.2DD1 and one M-414.3PD by Physik Instrumente, Karlsruhe, Germany), which allowed movement in XYZ-directions. The sample was moved with a controller that had a sequence programmed to its buttons ensuring a constant and repeatable chain of actions.

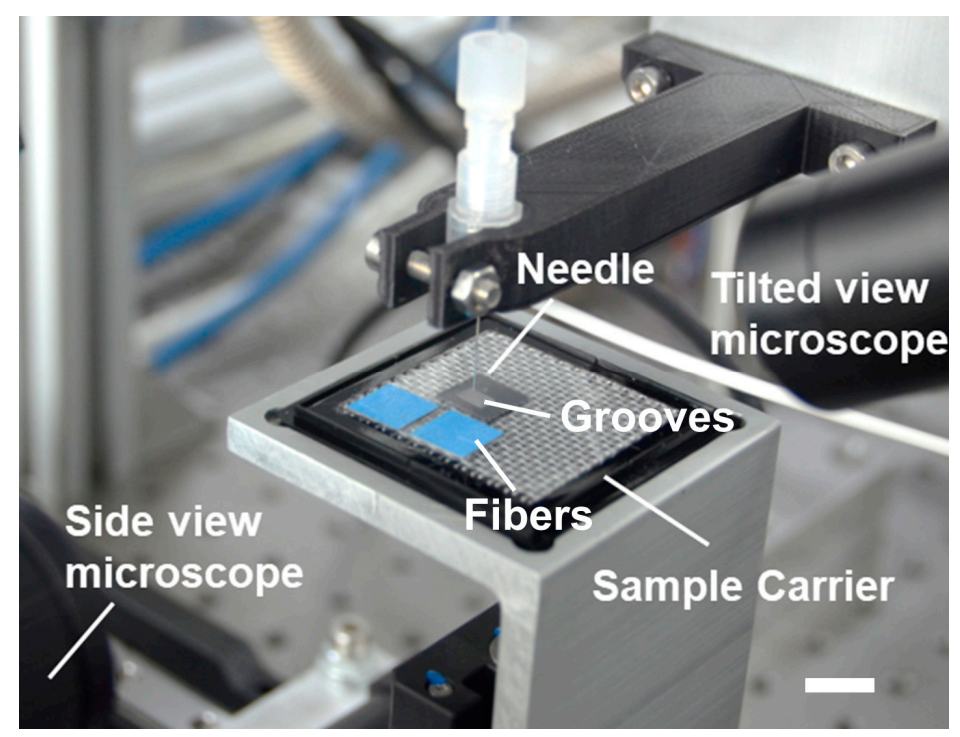

Figure 3. Robotic system for surface tension-based alignment of microfibers on hydrophilicsuperhydrophobic grooves. Scale bar: $10 \mathrm{~mm}$.

\section{Results}

\subsection{Simulations}

Surface tension-based alignment is based on the principle of surface energy minimization of the liquid medium, where the gradient of potential drives the microfiber to align with the microgrooves. To model the alignment process, we used Surface Evolver [32] to find the static equilibrium for the liquid medium by evolving the surface of the liquid using the gradient descent method. Surface Evolver breaks the surface of the liquid droplet into smaller elements, and minimizes the surface energy of each element, by optimizing the location of each vertex. Figure 4 illustrates the simulation of the alignment of microfiber on a hydrophilic-superhydrophobic groove. In the simulation, the size of the fiber is set to be $13 \mu \mathrm{m}$ in width and $4 \mathrm{~mm}$ in length, which matches the size of the glass fiber used in the experiments. The contact angle of the fiber is set to be $15^{\circ}$ because the fibers used in our experiments are made of glass, and the measured water contact angle on a glass substrate is $15^{\circ}$. The surface tension of the water is $72.8 \mathrm{mN} / \mathrm{m}$, and the volume of the droplet is set to be $10 \mathrm{~nL}$ which is the same as the volume used in the tests. The gravitational force of the glass fiber can be neglected because the size of the microfiber $(13 \mu \mathrm{m})$ is much smaller than the capillary length $(2.7 \mathrm{~mm})$ for water at standard temperature and pressure. The position difference between the release position of the fiber and the center of the groove is defined as the bias, which includes the bias in x-axis labeled as $\Delta x$, the bias in $y$-axis labeled as $\Delta y$ and the bias in z-axis labeled as $\Delta z$ as shown in Figure 4 . The center of the groove is labeled as "o" in Figure 4. 


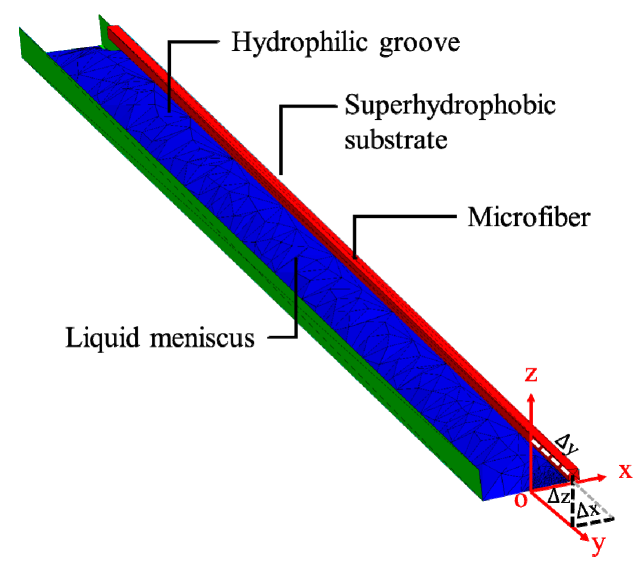

Figure 4. Surface Evolver model of the alignment of microfiber on a hydrophilic-superhydrophobic groove. The model includes three key elements: microfiber, hydrophilic-superhydrophobic groove, liquid meniscus.

The restoring force that drives the microfiber to align with the groove can be calculated by:

$$
\vec{F}(\Delta x, \Delta y, \Delta z)=-\nabla E(\Delta x, \Delta y, \Delta z)
$$

where $E$ is the surface energy of the liquid meniscus, and $\Delta x, \Delta y$, and $\Delta z$ are the biases along corresponding axes.

Figure 5 shows the surface energy and the restoring force of the water meniscus as the function of the $\mathrm{x}$-bias. The volume of the droplet was kept as $10 \mathrm{~nL}$ for all the simulations. The contact angle of water on the substrate was fixed at $155^{\circ}$, which is the same as the measured water contact angle of the superhydrophobic substrate in experiments. The contact angle in the groove was set to be $5^{\circ}$ which is the same as the estimation based on the measured contact angle of a water droplet in the groove. Figure 5 a shows the relation between the surface energy and the $x$-bias. The simulation results show that the surface energy of the water meniscus decreases as the fiber moves from its releasing position towards the center of the groove. The surface energy is minimized, and the water meniscus reaches its equilibrium state when the microfiber is aligned with the groove. Figure $5 b$ shows the restoring force as the function of $x$-bias. The simulation indicates the restoring force decreases as the bias decreases and the restoring force decreases to zero when the bias reaches zero, where the fiber is perfectly aligned with the groove.

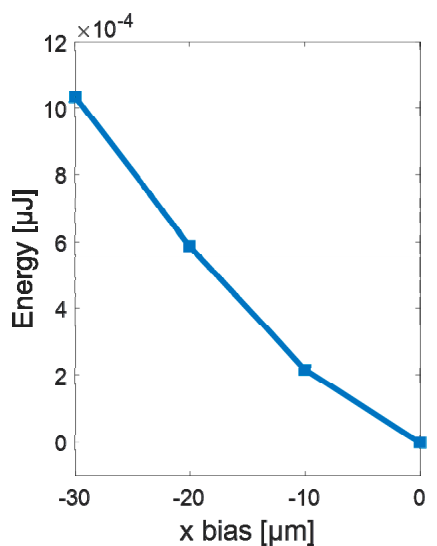

(a)

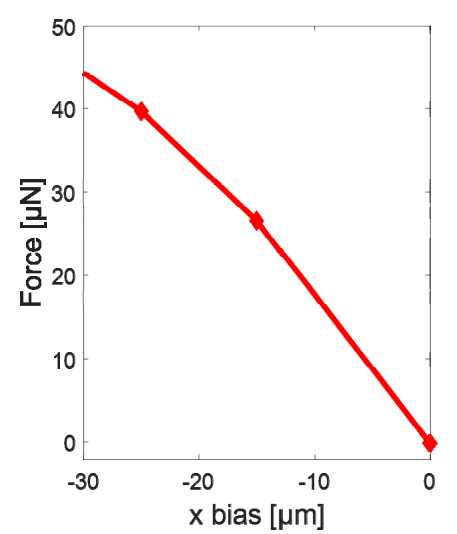

(b)

Figure 5. Surface energy and restoring force as the function of $x$-bias: (a) Surface energy of the water meniscus versus x-bias; (b) Restoring force driving alignment of microfiber on a hydrophilic-superhydrophobic groove versus $\mathrm{x}$-bias. 
To understand the influence of the wetting property of the groove on the alignment process, 5 sets of contact angles were simulated. The water contact angle of the groove varied from $0^{\circ}$ to $80^{\circ}$. The contact angle of water on the substrate was kept the same as $155^{\circ}$ for all the simulations. Figure 6 shows the surface energy of the water meniscus as the function of $x$-bias regarding different contact angles in the groove. The simulation indicates that the more hydrophobic the groove is, the more likely the alignment will fail. The reason is that the larger the contact angle is, the smaller the wetting contrast between the groove and the substrate, the flatter the energy curve (Figure 6a) becomes, and the flat energy curve leads to low restoring force for the alignment as shown in Figure $6 \mathrm{~b}$.

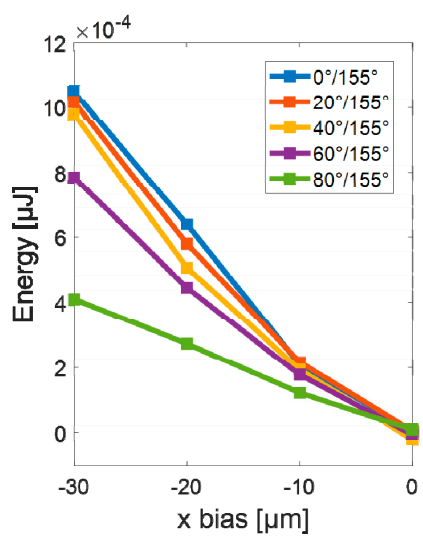

(a)

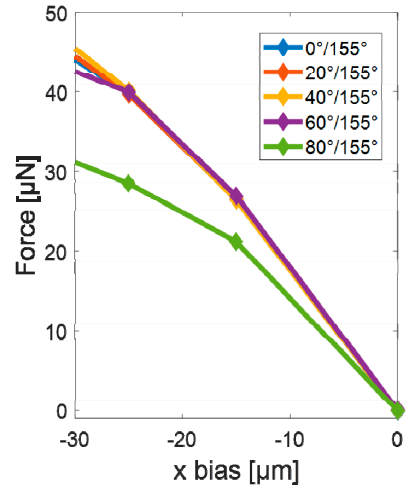

(b)

Figure 6. Surface energy and the restoring force as the function of $x$ bias regarding different wetting contrast of grooves: (a) Surface energy versus $x$-bias with a contact angle of the groove from $0^{\circ}$ to $80^{\circ}$; (b) Restoring force versus $x$-bias with a contact angle of the groove from $0^{\circ}$ to $80^{\circ}$.

\subsection{Experimental Results}

Series of experiments were carried out to investigate the proposed surface tension-based alignment method for microfibers. Figure 7 shows the sequences of picking up and releasing a $13 \mu \mathrm{m}$ wide and $4 \mathrm{~mm}$ long glass fiber. Firstly, the fiber is moved below a needle (Figure 7a); and next, the fiber is picked up by the needle (Figure $7 \mathrm{~b}$ ); after that, the fiber is transported to a $100 \mu \mathrm{m}$ wide and $4 \mathrm{~mm}$ long hydrophilic-superhydrophobic groove; then, the needle generates a $10 \mathrm{~nL}$ water droplet and the droplet is in contact with the hydrophilic groove with a water contact angle of $5^{\circ}$ forming a water meniscus (Figure 7c); finally, the fiber is released on the target groove and aligned with the groove (Figure 7d).
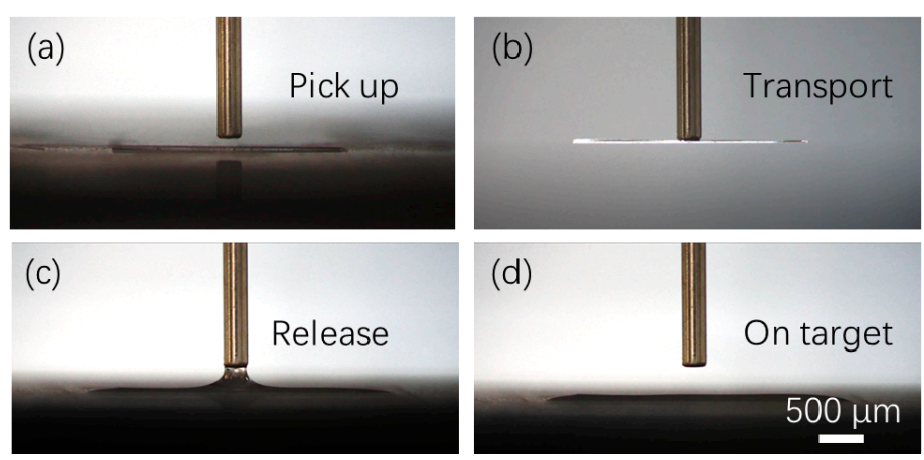

Figure 7. T Sequences of surface tension-based alignment of a glass fiber: $(\mathbf{a}, \mathbf{b})$ The needle picks up a microfiber and transport it to the target groove; (c) The needle generates a $10 \mathrm{~nL}$ water droplet and the droplet is in contact with the groove forming a water meniscus; (d) The water in the groove evaporates and the fiber is aligned with the groove. 
Surface tension-based alignment tests with different volume of the droplet ( $1 \mathrm{~nL}$ to $40 \mathrm{~nL}$ ) were carried out to find out the relationship between the volume and the success rate. In the experiments, the size of the groove is $4 \mathrm{~mm}$ (length) $\times 0.1 \mathrm{~mm}$ (width) $\times 0.035 \mathrm{~mm}$ (thickness). The size of the glass fiber is $4 \mathrm{~mm}$ (length) $\times 0.013 \mathrm{~mm}$ (diameter). A successful alignment refers to the fiber being aligned to the groove. Each test was repeated 5 times. The results were summarized in Table 1.

Table 1. Influence of volume of droplet on alignment.

\begin{tabular}{cc}
\hline Volume [nL] & Success Rate \\
\hline 40 & $100 \%$ \\
30 & $100 \%$ \\
20 & $100 \%$ \\
10 & $100 \%$ \\
5 & $80 \%$ \\
1 & $0 \%$ \\
\hline
\end{tabular}

Table 1 shows that the alignment process is very robust to the volume of the droplet and the alignment is reliable with the volume of droplet ranging from $10 \mathrm{~nL}$ to $40 \mathrm{~nL}$. When the volume of the droplet is less than $5 \mathrm{~nL}$, the alignment starts to fail mainly due to not enough liquid covering the groove which leads to dry contact and large friction force between the partially wetted groove and the fiber. The upper boundary of the volume is mainly determined by the amount of liquid that can be confined in the groove without spreading. In our case, the superhydrophobic coating plays a very import role in liquid confinement, and the liquid was well confined inside the groove until it reaches to $100 \mathrm{~nL}$.

We also carried out tests to study the influence of the bias on the alignment success rate. The size of the grooves used in the experiment is $4 \mathrm{~mm}$ (length) $\times 0.5 \mathrm{~mm}$ (width) $\times 0.035 \mathrm{~mm}$ (thickness) The size of the fiber is $4 \mathrm{~mm}$ (length) $\times 0.013 \mathrm{~mm}$ (diameter). Table 2 summarizes the results. The bias in Table 2 refers to the bias in x-axis as defined in Figure 4. The bias is ranging from $250 \mu \mathrm{m}$ to $50 \mu \mathrm{m}$, the volume of the droplet was kept the same $(40 \mathrm{~nL})$ for all the tests, and each test was repeated 5 times. The results indicate that the bias has little influence on the alignment process regarding the alignment success rate.

Table 2. Influence of bias on alignment.

\begin{tabular}{cc}
\hline Bias $[\mu \mathrm{m}]$ & Success Rate \\
\hline 250 & $100 \%$ \\
200 & $100 \%$ \\
150 & $100 \%$ \\
100 & $100 \%$ \\
50 & $100 \%$ \\
\hline
\end{tabular}

We have further investigated the relationship between the alignment accuracy and the width of the groove. Alignment accuracy can be described with the linear alignment error and angular alignment error. Linear alignment error is defined as the linear position difference between the final position of the fiber and the center of the groove labeled as " $\mathrm{o}$ " shown in Figure 4. Angular alignment error refers to the angular difference between the final position of the fiber and the edge of the groove. The width of the groove used in the experiments varied from $100 \mu \mathrm{m}$ to $500 \mu \mathrm{m}$. $10 \mathrm{~nL}$ of water is used in all the tests. Each test has been repeated at least 5 times. Figure 8a shows the angular alignment error as the function of the width of the groove. The $\mathrm{x}$-axis represents the width of the groove, and the $y$-axis represents the angular alignment error which consists of a mean of 5 repetitions with the standard derivation. The results indicate that the angular alignment error decreases as the width of the groove decreases. When the width of the groove is $100 \mu \mathrm{m}$ wide, the angular alignment error is less than $1^{\circ}$. It appears that the closer the width of the groove matches the diameter of the fiber, the better the fiber 
is parallel to the edge of the groove. Figure $8 \mathrm{~b}$ shows the linear alignment error as the function of the width of the groove. The results indicate that the linear alignment error decreases as the width of the groove decreases. When the groove is $100 \mu \mathrm{m}$ wide, the linear alignment error is around $15 \mu \mathrm{m}$. It appears that the closer the width of the groove matches the diameter of the fiber, the smaller the linear alignment error is.

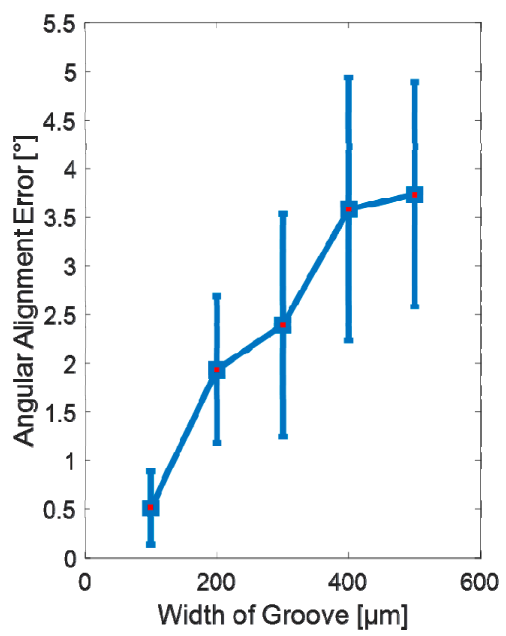

(a)

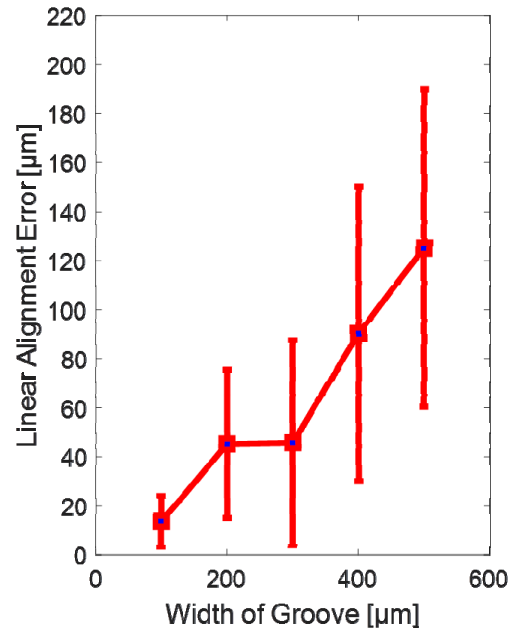

(b)

Figure 8. The influence of the width of the groove on the alignment accuracy: (a) Angular alignment error as the function of the width of the groove; (b) Linear alignment error as the function of the width of the groove.

To demonstrate the proposed surface tension-based alignment method can be used to construct more complex patterns and geometries with microfibers, we have fabricated hydrophilic-superhydrophobic grooves with different geometries, including T-shape, cross-shape, and parallel line-shape, and carried out the alignment tests. With the proposed method, the glass fibers can be distributed into a certain pattern according to the geometry of the hydrophilic-superhydrophobic grooves as shown in Figure 9.

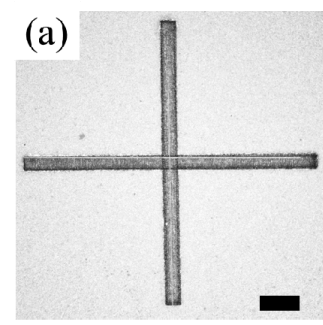

(b)

(c)
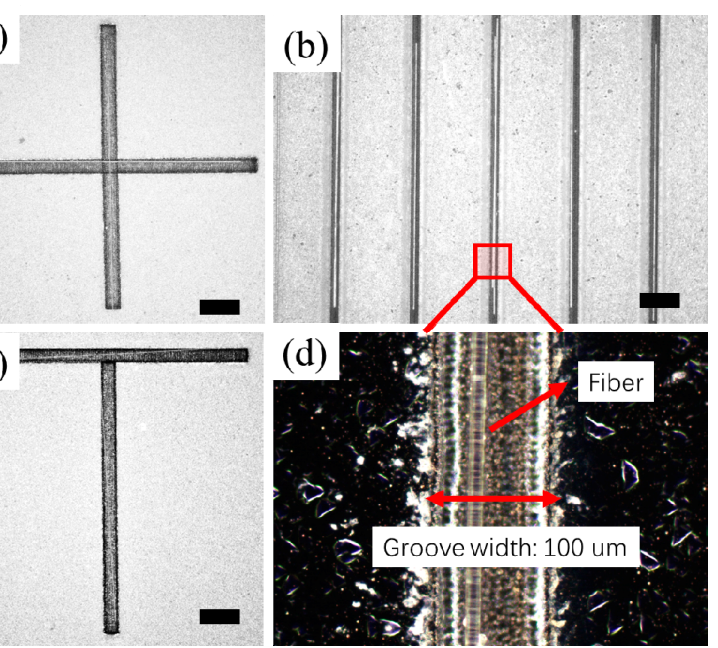

Figure 9. Demonstration of orderly distribution and alignment of microfibers on hydrophilic-superhydrophobic grooves: (a) Cross-shape distribution; (b) Parallel distribution; (c) T-shape distribution; (d) Zoomed image of a $100 \mu \mathrm{m}$ wide groove with a $13 \mu \mathrm{m}$ fiber inside. Scale bar: $400 \mu \mathrm{m}$. (Supplementary Video S1). 
The results show that microfibers can be distributed orthogonally (Figure 9a-c) and in parallel (Figure 9b). Figure 9d represents a zoomed image of a $13 \mu \mathrm{m}$ fiber being successfully aligned to a $100 \mu \mathrm{m}$ wide groove. A demonstration of the surface-tension based alignment of microfibers on the parallel hydrophilic-superhydrophobic grooved surface is shown in the supplementary video (Supplementary Video S1).

Figure 10 shows that 25 microfibers with a diameter of $13 \mu \mathrm{m}$ have been successfully aligned to its corresponding grooves with a width of $100 \mu \mathrm{m}$. This shows that the proposed surface tension-based alignment method has great potential to be applied to construct complex fiber-based microstructures.

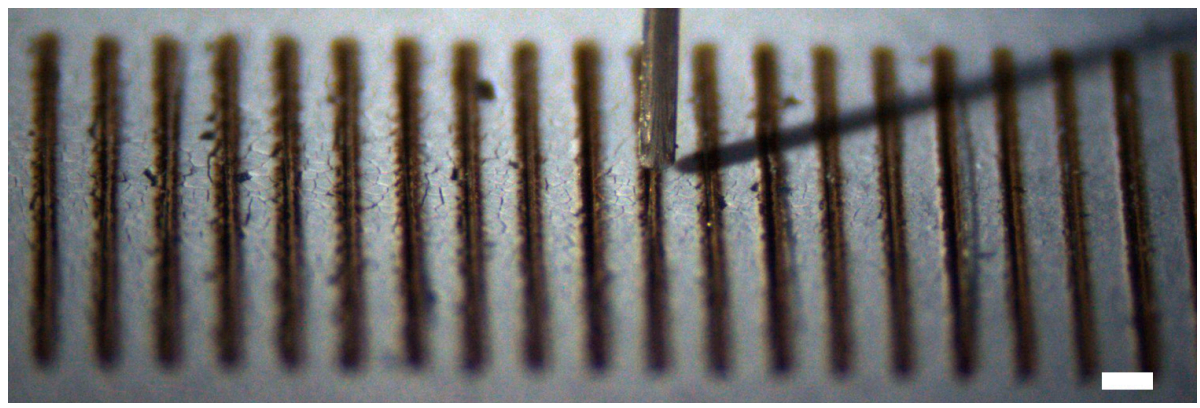

(a)

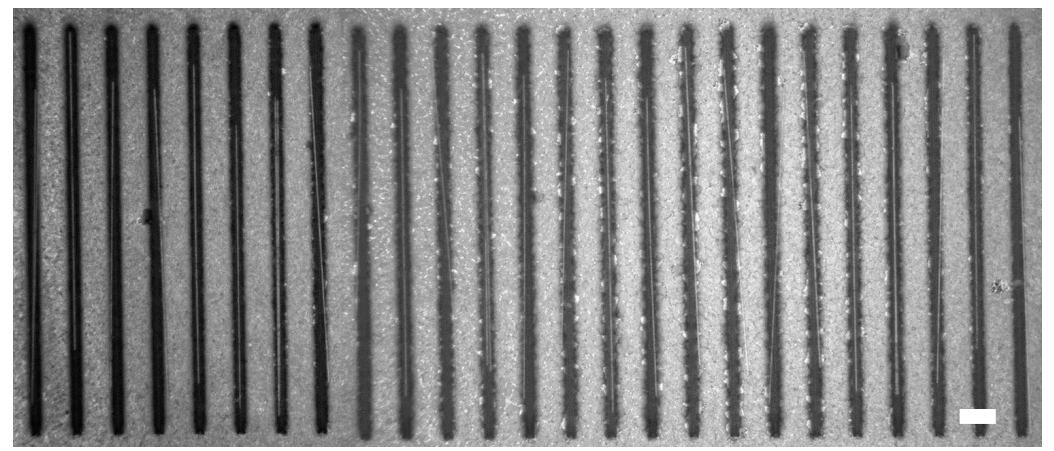

(b)

Figure 10. Alignment of 25 microfibers to parallel grooves: (a) Titled view of a fiber released to the corresponding hydrophilic-superhydrophobic groove; (b) Top view of 25 fibers aligned to the corresponding grooves. Scale bar: $200 \mu \mathrm{m}$.

\section{Discussions and Conclusions}

To sum up, the alignment accuracy is largely depending on the size difference between the groove and the microfiber, the better the size of the groove matches the size of the fiber, the higher the alignment accuracy will be achieved. In our experiments, the grooves were fabricated using an ultraviolet laser cutting machine and the minimum spot size is approximately $50 \mu \mathrm{m}$, which limited the capability of fabricating grooves with the width less than $100 \mu \mathrm{m}$. The width of the groove could be further decreased to several to tens of micrometers using a picosecond laser cutting technique to match the size of the microfiber. The proposed alignment methods will be extended by using sacrificial substrate for potential applications in fabrication of advanced fiber-enhanced functional materials. In our experiments, we used glass fibers, but this proposed surface tension-based alignment method might be also applied to the microfibers of other materials. Although we only tested water as the medium for the alignment, other low surface tension liquid may also work if the liquid can be confined inside the groove. The alignment with water normally takes tens of milliseconds, regarding to the liquid medium of higher evaporation rate, such as ethanol, if the evaporation of the liquid is slower than the alignment, the alignment should also work.

This paper proposes a surface tension-based method to align and orderly distribute microfibers. A theoretical model was developed to simulate the alignment of the microfiber and analyze the 
driven force for the alignment of microfibers. The simulation shows the larger the wetting contrast of the groove, the larger the restoring force for alignment. We designed and fabricated hydrophilic-superhydrophobic grooved surfaces. We demonstrated that the microfibers can self-align to the hydrophilic-superhydrophobic grooves with different geometries. We further studied the influence of the volume of the droplet and the bias on the alignment success rate. The results indicate that the process can tolerate large variations of the bias and the volume unless the volume is not enough to cover the groove. Furthermore, we investigated the influence of the width of the grooves on the alignment accuracy and verified that the alignment accuracy increases as the width of the groove decrease. The proposed method has great potential in construction of complex microstructures using microfibers.

Supplementary Materials: The following are available online at http:/www.mdpi.com/2072-666X/11/11/973/s1, Video S1: Surface Tension-based Alignment of Microfibers on Hydrophilic-superhydrophobic Grooved Surface.

Author Contributions: B.C. conceived and designed the work; J.J. performed the experiments; B.C. analyzed the data; B.C. wrote the paper. Q.Z. contributed to the evaluation and the manuscript writing. All authors have read and agreed to the published version of the manuscript.

Funding: This research was funded by the National Natural Science Foundation of China (grant no. 61703255) and the Academy of Finland (Grant No. 296250).

Acknowledgments: The authors would like to thank Yongshuai $\mathrm{Hu}$ for collaborations on fabrication of hydrophilic-superhydrophobic grooved surfaces.

Conflicts of Interest: The authors declare no conflict of interest.

\section{References}

1. Greenhall, J.; Guevara Vasquez, F.; Raeymaekers, B. Ultrasound directed self-assembly of user-specified patterns of nanoparticles dispersed in a fluid medium. Appl. Phys. Lett. 2016, 108, 103103. [CrossRef]

2. Gupta, P.; Rajput, M.; Singla, N.; Kumar, V.; Lahiri, D. Electric field and current assisted alignment of CNT inside polymer matrix and its effects on electrical and mechanical properties. Polymer 2016, 89, 119-127. [CrossRef]

3. Fischer, J.E.; Zhou, W.; Vavro, J.; Llaguno, M.C.; Guthy, C.; Haggenmueller, R.; Casavant, M.J.; Walters, D.E.; Smalley, R.E. Magnetically aligned single wall carbon nanotube films: Preferred orientation and anisotropic transport properties. J. Appl. Phys. 2003, 93, 2157-2163. [CrossRef]

4. Manoj Kumar, R.; Sharma, S.K.; Manoj Kumar, B.V.; Lahiri, D. Effects of carbon nanotube aspect ratio on strengthening and tribological behavior of ultra high molecular weight polyethylene composite. Compos. Part A Appl. Sci. Manuf. 2015, 76, 62-72. [CrossRef]

5. Wang, Q.; Dai, J.; Li, W.; Wei, Z.; Jiang, J. The effects of CNT alignment on electrical conductivity and mechanical properties of SWNT/epoxy nanocomposites. Compos. Sci. Technol. 2008, 68, 1644-1648. [CrossRef]

6. Ramos, J.A.; Esposito, L.; Kortaberria, G.; D'Arlas, B.F.; Zalakain, I.; Goyanes, S.; Mondragon, I. Electric field alignment of multi-walled carbon nanotubes through curing of an epoxy matrix. J. Nanostruct. Polym. Nanocompos. 2012, 8, 89-93.

7. Pang, C.; Lee, G.Y.; Kim, T.I.; Kim, S.M.; Kim, H.N.; Ahn, S.H.; Suh, K.Y. A flexible and highly sensitive strain-gauge sensor using reversible interlocking of nanofibres. Nat. Mater. 2012, 11, 795-801. [CrossRef]

8. Li, D.; Wang, Y.; Xia, Y. Electrospinning of polymeric and ceramic nanofibers as uniaxially aligned arrays. Nano Lett. 2003, 3, 1167-1171. [CrossRef]

9. Liu, Y.; Zhang, X.; Xia, Y.; Yang, H. Magnetic-field-assisted electrospinning of aligned straight and wavy polymeric nanofibers. Adv. Mater. 2010, 22, 2454-2457. [CrossRef]

10. Arras, M.M.L.; Grasl, C.; Bergmeister, H.; Schima, H. Electrospinning of aligned fibers with adjustable orientation using auxiliary electrodes. Sci. Technol. Adv. Mater. 2012, 13, 035008. [CrossRef]

11. Subramanian, A.; Krishnan, U.M.; Sethuraman, S. Fabrication of uniaxially aligned 3D electrospun scaffolds for neural regeneration. Biomed. Mater. 2011, 6, 025004. [CrossRef]

12. Brown, T.D.; Dalton, P.D.; Hutmacher, D.W. Direct writing by way of melt electrospinning. Adv. Mater. 2011, 23, 5651-5657. [CrossRef] 
13. Yu, Y.; Hua, S.; Yang, M.; Fu, Z.; Teng, S.; Niu, K.; Zhao, Q.; Yi, C. Fabrication and characterization of electrospinning/3D printing bone tissue engineering scaffold. RSC Adv. 2016, 6, 110557-110565. [CrossRef]

14. Costantini, M.; Testa, S.; Mozetic, P.; Barbetta, A.; Fuoco, C.; Fornetti, E.; Tamiro, F.; Bernardini, S.; Jaroszewicz, J.; Święszkowski, W.; et al. Microfluidic-enhanced 3D bioprinting of aligned myoblast-laden hydrogels leads to functionally organized myofibers in vitro and in vivo. Biomaterials 2017, 131, 98-110. [CrossRef] [PubMed]

15. Saketi, P.; Treimanis, A.; Fardim, P.; Ronkanen, P.; Kallio, P.; Hirvonen, J.; Kallio, P.; Sun, T.; Li, X.; Shi, Q.; et al. Electrospinning and electrospun nanofibers: Methods, materials, and applications. Materials 2019, 6, 2611.

16. Hirvonen, J.; Von Essen, M.; Kallio, P. Automated microrobotic manipulation of paper fiber bonds. In Proceedings of the IEEE International Conference on Intelligent Robots and Systems, Hamburg, Germany, 17 December 2015.

17. Von Essen, M.; Hirvonen, J.; Kuikka, S.; Kallio, P. Towards fully automated pick and place operations of individual natural fibers. In Proceedings of the 2013 International Conference on Manipulation, Manufacturing and Measurement on the Nanoscale, 3M-NANO 2013-Conference Proceedings, Suzhou, China, 26-30 August 2013.

18. Sun, T.; Li, X.; Shi, Q.; Wang, H.; Huang, Q.; Fukuda, T. Microfluidic Spun Alginate Hydrogel Microfibers and Their Application in Tissue Engineering. Gels 2018, 4, 38. [CrossRef]

19. Zhang, X.; Weng, L.; Liu, Q.; Li, D.; Deng, B. Facile fabrication and characterization on alginate microfibres with grooved structure via microfluidic spinning. R. Soc. Open Sci. 2019, 6, 181928. [CrossRef] [PubMed]

20. Jeong, Y.H.; Lee, J. Fabrication of microfiber patterns with ivy shoot-like geometries using improved electrospinning. Materials 2016, 9, 266. [CrossRef] [PubMed]

21. Chang, B.; Zhou, Q.; Wu, Z.; Liu, Z.; Ras, R.; Hjort, K. Capillary Self-Alignment of Microchips on Soft Substrates. Micromachines 2016, 7, 41. [CrossRef]

22. Chang, B.; Sariola, V.; Jääskeläinen, M.; Zhou, Q. Self-alignment in the stacking of microchips with mist-induced water droplets. J. Micromech. Microeng. 2011, 21, 015016. [CrossRef]

23. Sariola, V.; Jääskeläinen, M.; Zhou, Q. Hybrid microassembly combining robotics and water droplet self-alignment. IEEE Trans. Robot. 2010, 26, 965-977. [CrossRef]

24. Chang, B.; Liu, H.; Ras, R.H.A.; Zhou, Q. Capillary Transport of Miniature Soft Ribbons. Micromachines 2019, 10, 684. [CrossRef]

25. Chang, B.; Zhu, Z.; Koverola, M.; Zhou, Q. Laser-Assisted Mist Capillary Self-Alignment. Micromachines 2017, 8, 361. [CrossRef] [PubMed]

26. Mastrangeli, M.; Zhou, Q.; Sariola, V.; Lambert, P. Surface tension-driven self-alignment. Soft Matter 2017, 13, 304-327. [CrossRef] [PubMed]

27. Ito, Y.; Fukushima, T.; Lee, K.W.; Tanaka, T.; Koyanagi, M. Capillary Self-Assembly for 3D Heterogeneous System Integration and Packaging. MRS Adv. 2016, 1, 2355-2366. [CrossRef]

28. Ito, Y.; Fukushima, T.; Kino, H.; Lee, K.W.; Tanaka, T.; Koyanagi, M. Impact of Chip-Edge Structures on Alignment Accuracies of Self-Assembled Dies for Microelectronic System Integration. J. Microelectromech. Syst. 2016, 25, 91-100. [CrossRef]

29. Lambert, P.; Mastrangeli, M.; Valsamis, J.B.; Degrez, G. Spectral analysis and experimental study of lateral capillary dynamics for flip-chip applications. Microfluid. Nanofluid. 2010, 9, 797-807. [CrossRef]

30. Arutinov, G.; Smits, E.C.P.; Albert, P.; Lambert, P.; Mastrangeli, M. In-plane mode dynamics of capillary self-alignment. Langmuir 2014, 30, 13092-13102. [CrossRef]

31. Zhou, Q.; Sariola, V.; Chang, B. Towards hybrid assembly of RFIDs. In Proceedings of the Smart Systems Integration 2010 - 4th European Conference and Exhibition on Integration Issues of Miniaturized Systems-MEMS, MOEMS, ICs and Electronic Components, Como, Italy, 23-24 March 2010.

32. Brakke, K.A. The Surface Evolver. Exp. Math. 1992, 1, 141-165. [CrossRef]

Publisher's Note: MDPI stays neutral with regard to jurisdictional claims in published maps and institutional affiliations. 